\title{
Hepatic involvement in granulomatosis with polyangiitis - diagnostic difficulties
}

\author{
Anna Masiak ${ }^{1}$, Anna Drobińska², Zbigniew Zdrojewski ${ }^{1}$ \\ ${ }^{1}$ Clinic of Internal Medicine, Connective Tissue Diseases, and Geriatrics, Medical University of Gdansk, Poland \\ ${ }^{2}$ Clinic of Gastroenterology and Hepatology, Medical University of Gdansk, Poland
}

\begin{abstract}
We report the case of a 57-year-old Caucasian man who presented with dry cough, haemoptysis, fever, lung nodules, erythrocyturia, and acute hepatitis. After a lung biopsy, the patient was diagnosed with granulomatosis with polyangiitis. The diagnosis was supported by the presence of antiproteinase-3 anti-neutrophil cytoplasmic antibodies. The most common causes of liver damage are excluded.

The patient was treated with prednisone and cyclophosphamide, which resulted in remission of chest CT findings and improvement in liver function tests. During the flare, new lung infiltrations as well as elevation of liver enzymes were present. Treatment with rituximab resulted in complete clinical and radiological remission and normalisation of liver function tests. What makes this case worth reporting is the rare liver involvement in the GPA. After exclusion of coexistence of autoimmune liver disease and drug-induced liver lesion, organ involvement was supported by the reversion of abnormal LFT after the immunosuppression of GPA.
\end{abstract}

Key words: granulomatosis with polyangiitis, differential diagnosis, hepar involvement.

\section{Introduction}

Granulomatosis with polyangiitis (GPA) is an anti-neutrophil cytoplasmic autoantibody (ANCA)-associated small and medium vessel systemic vasculitis (AAV) characterised primarily by necrotising granulomatosis of the upper and lower respiratory tract and by glomerulonephritis [1]. However, GPA can principally affect any organ in a vasculitic or granulomatous disease pattern. Elevated liver function tests (LFT) are quite often present in AAV patients. The most common clinical presentation is a mild lesion due to drug exposure, especially NSAID and antibiotic. The actual incidence of liver involvement in GPA is unclear [2]. In clinical practice, even after careful exclusion of previous treatment with potentially hepatotoxic drugs or coincident viral hepatitis, the question remains whether to classify the patient as having a primary liver disease with associated autoimmune or as having liver disease as a manifestation of generalised connective tissue disease. Here we present a patient with GPA and liver function test abnormalities, and discuss diagnostic and therapeutic difficulties.

\section{Case report}

A 57-year-old Caucasian man was admitted to hospital with dry cough, haemoptysis, fever, night sweats, arthralgia, and recurrent conjunctivitis for four months. Laboratory investigation showed mild anaemia, leukocytosis, thrombocytosis, elevated acute phase markers, and liver function tests (aspartate aminotransferase, AST $<2 \times$ upper normal limit - ULN); alanine aminotransferase, ALT < $2 \times$ ULN; alkaline phosphatase, ALP $4 \times$ ULN; gamma-glutamyltransferase, GGT $12 \times$ ULN). A urine analysis was negative for glycosuria and proteinuria, while urine microscopy revealed three to five red blood cells per high power field and the presence of occasional dysmorphic red blood cells. The laboratory results are shown in Table I. 
Table I. Laboratory test at the beginning, during exacerbation, in remission, during relapse and after rituximab

\begin{tabular}{|c|c|c|c|c|c|}
\hline $\begin{array}{l}\text { Laboratory test } \\
\text { [normal range] }\end{array}$ & $\begin{array}{l}\text { At the } \\
\text { beginning }\end{array}$ & $\begin{array}{c}\text { During exacerbation } \\
\text { ( } 6 \text { months after the beginning } \\
\text { of treatment) }\end{array}$ & First remission & Relapse & $\begin{array}{l}\text { Second } \\
\text { remission }\end{array}$ \\
\hline $\begin{array}{l}\text { White blood cells, WBC } \\
{\left[2-7 \times 10^{9} / 1\right]}\end{array}$ & 12.5 & 10.4 & 14.5 & 13.5 & 5.2 \\
\hline $\begin{array}{l}\text { Haemoglobin, } \\
\text { Hgb [13.0-17.0 g/dl] }\end{array}$ & 9.8 & 13.4 & 15.1 & 14.4 & 14.5 \\
\hline $\begin{array}{l}\text { Platelets, } \\
\text { PLT }\left[150-410 \times 10^{9} / l\right]\end{array}$ & 870 & 330 & 203 & 442 & 254 \\
\hline $\begin{array}{l}\text { Creatinine } \\
{[0.73-1.18 \mathrm{mg} / \mathrm{dl}]}\end{array}$ & 0.68 & 0.8 & 1.1 & 1.02 & 0.87 \\
\hline $\begin{array}{l}\text { Erythrocyte sedimentation } \\
\text { rate, ESR [2-20 } \mathrm{mm} / \mathrm{h}]\end{array}$ & 119 & 75 & 31 & 40 & 16 \\
\hline $\begin{array}{l}\text { C-reactive protein, CRP } \\
{[0.0-5.0 \mathrm{mg} / \mathrm{l}]}\end{array}$ & 190 & 116 & 15 & 98.3 & 5.3 \\
\hline $\begin{array}{l}\text { Alanine aminotransferase ALT } \\
{[<55 \mathrm{U} / \mathrm{l}]}\end{array}$ & 105 & 133 & 40 & 44 & 15 \\
\hline $\begin{array}{l}\text { Aspartate aminotransferase, } \\
\text { AST [5-34 U/l] }\end{array}$ & 65 & 66 & 15 & 36 & 20 \\
\hline $\begin{array}{l}\gamma \text {-glutamyl transpeptidase, GGT } \\
{[12-64 \mathrm{U} / \mathrm{l}]}\end{array}$ & 809 & 1020 & 420 & 387 & 66 \\
\hline $\begin{array}{l}\text { Alkaline phosphatase, } \\
\text { ALP [40-150 U/I] }\end{array}$ & 558 & 244 & 158 & 178 & 50 \\
\hline Serum total protein [64-83 G/l] & 84 & 72 & 73 & 77 & $\mathrm{np}$ \\
\hline Albumin $[35.0-50 \mathrm{~g} / \mathrm{l}]$ & 21 & 32 & 36 & 35 & $\mathrm{np}$ \\
\hline$\gamma$-globulins [11.1-18.8\%] & 15.2 & 11.7 & 8.7 & 12.6 & $\mathrm{np}$ \\
\hline cANCA [<1:40] & $1: 640$ & $\mathrm{np}$ & negative & $1: 160$ & negative \\
\hline PR3-ANCA [<20 RU/ml] & $>200$ & $>200$ & 19.2 & $>200$ & 21.4 \\
\hline $\operatorname{lgG}[5.4-18.22 \mathrm{~g} / \mathrm{l}]$ & $\mathrm{np}$ & 9.4 & $\mathrm{np}$ & 7.05 & 6.02 \\
\hline $\operatorname{lgG} 4[0.03-2.01 \mathrm{~g} / 1]$ & np & 3.8 & np & 2.31 & $n p$ \\
\hline
\end{tabular}

np-not performed

Based on serological tests, active hepatitis B, hepatitis $C$, and human immunodeficiency virus infection were excluded. CT of the chest revealed multiple small bilateral pulmonary nodules, also with cavitation and lymphadenopathy (Fig. 1). Abdomen CT scan revealed only lymphadenopathy of hepatic recess. Mycobacterial or fungal infection were excluded (negative cultures of bronchoalveolar lavage, negative Quantiferon TB gold test). Biopsy of the right lung nodule presented granulomatous inflammation with necrotising granulomas of lung tissue, consistent with GPA.

The diagnosis was supported by the presence of both immunofluorescence assay (cANCA 1 : 640) and Elisa anti-proteinase-3 anti-neutrophil cytoplasmic antibodies (ANCA-PR3 > $200 \mathrm{RU} / \mathrm{ml}$, normal $<20 \mathrm{RU} / \mathrm{ml}$ ). ANCA immunoblot was positive for anti-PR3 antibodies. Renal biopsy was not performed. The patient was started on steroids (methylprednisolone $3 \times 1000$ mg IV, sub- sequent prednisone $1 \mathrm{mg} / \mathrm{kg} /$ day) and cyclophosphamide according to EUVAS recommendation $(15 \mathrm{mg} / \mathrm{kg}$ IV, max $1.2 \mathrm{~g}$, every two weeks for the first three pulses, followed by infusions every three weeks for the next six pulses) [3]. Initially the patient's clinical condition gradually improved, but after six months of treatment his clinical state deteriorated. He had fever, cough, and general malaise. Lung CT revealed progression of pulmonary infiltrations. Elevation of LFT (AST $2 \times$ ULN, ALT $2.5 \times$ ULN, ALP $1.5 \times$ ULN, GGT $16 \times$ ULN) and CRP $(116 \mathrm{mg} / \mathrm{l})$ was present in laboratory results. Due to sustained increase in LFT, a screening for viral and autoimmune hepatic diseases was performed. Active viral infection caused by cytomegalovirus, Epstein-Barr virus, and hepatitis $B$ and $C$ were excluded. Antibodies against smooth muscle (ASMA) were tested via indirect immunofluorescence assay according to the manufacturer's instructions. Additionally, ANA HEP-2, AMA-M2, and antibodies against 


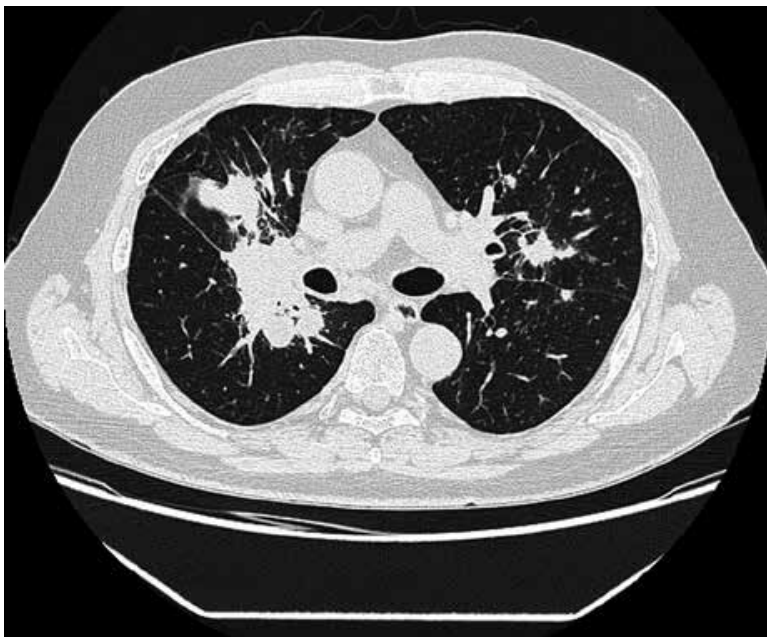

Fig. 1. Chest CT: Fibrotic and nodular changes in the right lung. Regions of consolidation with cavitation. Bilateral multiple nodules.

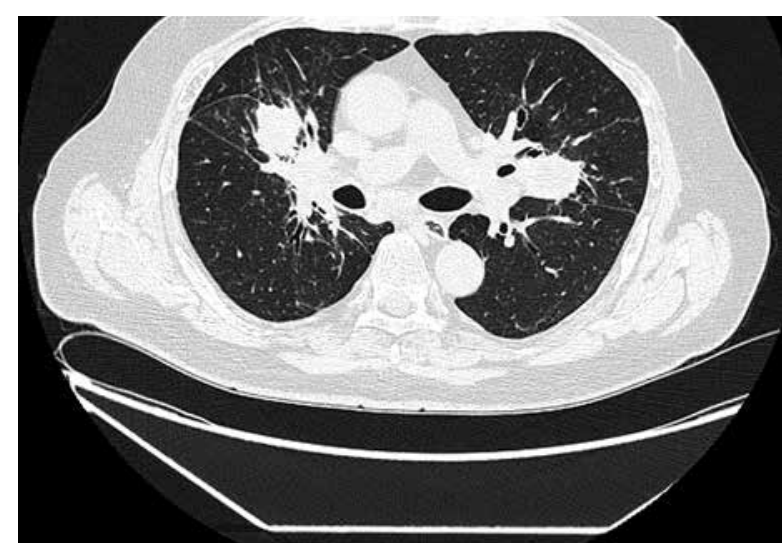

Fig. 2. Chest CT: New infiltrations typical for GPA.



Fig. 3. Chest CT after rituximab treatment: Significant regression of fibrous and nodular lesions.
Sp100, PML, gp210, LKM-1, LC-1, and SLA/LP were analysed by line-blot immunoassay (EUROIMMUN, Lübeck, Germany) and gave negative results. Magnetic resonance cholangiopancreatography (MRCP) showed no changes typical for primary sclerosing cholangitis. Cyclophosphamide therapy was continued for another three months to a total dose of CYC $13 \mathrm{~g}$, and finally the patient went into clinical remission. Azathioprine was started for remission maintenance. LFT improved but remained above the upper limit of normal (GGT > $6 \times U L N$, ALP $1.3 \times$ ULN, AST and ALT normal). After another four months of treatment with steroids (prednisone $10 \mathrm{mg}$ ) and azathioprine ( $2 \mathrm{mg} / \mathrm{kg} / \mathrm{day}$ ) he was hospitalised due to cough, haemoptysis, hoarseness, and fever. CT of lung revealed new infiltrations typical for GPA (Fig. 2).

Liver enzymes (mostly GGT $6 \times$ ULN) as well as CRP were elevated. Proteinuria was present in urine test. ANCA-PR3 antibodies were present in high titre (Table I). The patient was given pulses of cyclophosphamide every two weeks. Then, due to persistent symptoms of active disease, it was decided to change treatment to rituximab given in total dose of $375 \mathrm{mg} / \mathrm{m}^{2}$ of body area, once a week for four infusions. Activity of the disease was assessed six months after therapy, and complete remission was achieved (BVAS =0) (Fig. 3). LFT normalised for the first time since the initial diagnosis of GPA and the start of immunosuppressive therapy. He is treated with azathioprine (2 mg/kg/day) and prednisone (5 mg/ day) for remission maintenance. Twelve months after therapy he is still in clinical remission and his laboratory tests are normal.

\section{Discussion}

Liver involvement in rheumatic diseases, like systemic lupus erythematosus (SLE), primary Sjögren's syndrome, systemic sclerosis, or rheumatoid arthritis has often been reported, most commonly in the form of liver function test (LFT) biochemical changes with predominant cholestatic or hepatocellular patterns [Review in 4]. Also, an association of connective tissue diseases with autoimmune hepatitis (AlH), primary biliary cirrhosis (PBC), and primary sclerosing cholangitis (PSC) is well known [5]. The association between autoimmune liver diseases and small vessel vasculitis is rare but possible [6]. The incidence of liver involvement in GPA is rarely described, mainly in case series [7, 8]. Among the 180 GPA patients of the WGET study [9], no hepatic involvement was described. The Se.Pri.Va. study group found elevated liver enzymes in $8-11 \%$ of 36 GPA patients and Pavone et al. [10] in 2-25\% of AAV patients at the time of diagnosis. 
Willeke et al. [11] demonstrated that the liver is frequently affected (49.4\%) in patients with active GPA when affection is mirrored by liver test abnormalities. They also detected an association of increased LFT in GPA patients with the BVAS (Birmingham Vasculitis Activity Score), severe disease, and with pulmonary or pulmonary-renal involvement (increased values for $\gamma$-GT in GPA patients correlated with the BVAS and were associated with pulmonary involvement, pulmonary-renal syndrome, and a longer time to remission). The biochemical picture during active disease revealed both a cholestatic pattern (i.e. increased $\gamma$-GT and ALP) as well as a hepatocellular pattern (with increased ALT and AST). No concomitant autoimmune liver disease and no association with potential hepatotoxic drugs was observed [11]. There are also case reports of hepatitis in a GPA patient as a rare adverse effect of CYC, which responded to CYC withdrawal [12]. Literature data suggest that physicians should be aware of the possible occurrence of increased LFT in the active state of the disease. However, the results reported by Willeke et al. [11] indicate that increased LFT should not be a reason to rule out the use of cyclophosphamide. The authors have shown that abnormal LFT normalises during induction therapy in most cases despite the use of the potentially hepatotoxic cyclophosphamide or other DMARDS [11]. The diagnosis of hepatic involvement in GPA could be assumed after exclusion of other causes of hepatitis. Acute or progressing liver involvement is generally related to viral hepatitis reactivation, concomitant autoimmune liver disease, or drug-induced alteration. It is important to screen patients for HBV and HCV infection, to avoid life-threatening reactivations.

As suggested by Selmi et al. [13], autoimmune hepatic diseases should be considered once hepatic and/ or cholestatic biochemical profiles persist and are not clearly explained by liver involvement of a GPA or other rheumatic disease. A biopsy, as an invasive procedure, should only be performed in the presence of challenging diagnoses [5]. In our case, an adverse drug effect of cyclophosphamide could be excluded, since the lesions had already been present before CYC therapy. Also, other drug-induced liver damage was highly unlikely because liver dysfunction and the elevation of hepatic enzymes persisted after the discontinuation of antibiotics and NSAIDs. The patient denied alcohol consumption. Viral hepatitis was ruled out considering that serology for acute HBV and HCV infections were negative. The possibility of ischaemic hepatitis in a patient without previous heart condition history was unlikely. Elevation of LFT was associated with high activity of GPA, which also supports the close relationship between liver involvement and GPA in our patient.
Autoimmune hepatitis (AlH) cannot be completely ruled out because $10 \%$ of these cases may present with negative antinuclear antibodies, anti-smooth muscle antibodies, and anti-liver kidney microsomal antibodies. It should also be noted that the immunological tests were performed during immunosuppressive therapy, which could have affected the results. Furthermore, ANCAs are positive in $65 \%$ to $95 \%$ of patients with $\mathrm{AlH}$. However, they are usually of the perinuclear type. Our patient had CANCA, which is known to be typical for GPA.

IgG4-related disease (IgG4-RD) is a recently recognised multiorgan fibroinflammatory disease [14]. Patients with IgG4-RD typically have increased serum IgG4 levels, tissue infiltration with abundant lgG4-positive plasma cells in a background of dense storiform fibrosis, and multiple organ involvement and response to corticosteroid treatment. Many manifestations of hepatobiliary involvement by IgG4-RD have been described, including IgG4-associated sclerosing cholangitis (IgG4-SC), IgG4 hepatopathy, sclerosing cholecystitis, and IgG4-associated inflammatory pseudotumours. In many cases of IgG4-SC, diagnosis can be made by a combination of characteristic biliary images (MRCP, ERCP, and EUS), increased serum levels of IgG4, coexistence of other organ involvements, and characteristic histopathological features [15]. Elevated serum IgG4 along with GGT and ALP suggested IgG4-SC. The MRI image was not typical for IgG4SC in our patient. There was no other organ involvement. Literature data indicate, however, that IgG4 can also be increased in systemic vasculitis [16].

\section{Conclusions}

Physicians should be aware of the possible occurrence of increased LFT in the active state of vasculitis. The diagnosis of hepatic involvement in GPA could be assumed after exclusion of other causes of hepatitis. A biopsy, as an invasive procedure, should only be performed in the presence of challenging diagnoses. Increased LFT should not be a reason to rule out the use of cyclophosphamide if indicated according to active organ- or life-threatening vasculitis.

The authors declare no conflict of interest.

\section{References}

1. Jennette JC. Nomenclature and classification of vasculitis: lessons learned from granulomatosis with polyangiitis. Clin Exp Immunol 2011; 164 (Suppl. 1): 7-10.

2. Goritsas C, Paissios NP, Trigidou R, Delladetsima J. Hepatic involvement in Wegener's granulomatosis: a case report. J Med Case Rep 2010; 4: 9. 
3. Yates $M$, Watts RA, Bajemna IM, et al. EULAR-EDTA recommendations for the management of ANCA-associated vasculitis. Ann Rheum Dis 2016; 75: 1583-1594.

4. Soultati A, Dourakis S. Hepatic manifestations of autoimmune rheumatic diseases. Ann Gastroenterol 2005; 18: 309-324.

5. De Santis M, Crotti C, Selmi C. Liver abnormalities in connective tissue diseases. Best Pract Res Clin Gastroenterol 2013. 27: 543-551.

6. Tovoli F, Vannini A, Fusconi M, et al. Autoimmune liver diseases and small-vessel vasculitis: four case reports and review of the literature. Ann Hepatol 2014; 13: 136-141.

7. Shah IA, Holstege A, Riede UN. Bioptic diagnosis of Wegener's granulomatosis in the absence of vasculitis and granulomas. Pathol Res Pract 1984; 178: 407-415.

8. Den Bakker MA, Tangkau PL, Steffens TW, et al. Rupture of a hepatic artery aneurysm caused by Wegener's granulomatosis. Pathol Res Pract 1997; 193: 61-66.

9. Seo P, Miny YI, Holbrook JK, et al. Damage caused by Wegener's granulomatosis and its treatment: prospective data from the Wegener's Granulomatosis Etanercept Trial (WGET). Arthritis Rheum 2005; 52: 2168-2178.
10. Pavone L, Grasselli C, Chierici E, et al. Outcome and prognostic factors during the course of primary small-vessel vasculitides. J Rheumatol 2006; 33: 1299-1306.

11. Willeke P, Schlüter B, Limani A, et al. Liver involvement in ANCA-associated vasculitis. Clin Rheumatol 2016; 35: 387-394.

12. Patel A, Patel S, Patel S, et al. Cyclophosphamide Therapy in Granulomatous Hepatitis: Cure or Culpit? Am J Therapeut 2009; 16: 367-370.

13. Selmi C, De Santis M, Gershwin ME. Liver involvement in subjects with rheumatic disease. Arthritis Res Ther 2011; 13: 226.

14. Umehara H, Okazaki K, Masaki Y, et al. Comprehensive diagnostic criteria for IgG4-related disease (IgG4-RD). Mod Rheumatol 2012; 22: 21

15. Graham R, Smyrk T, Chari S, et al. Isolated IgG4-related sclerosing cholangitis: a report of 9 cases. Human Pathol 2014; 45: 1722-1729.

16. Perez AR, Martínez C, Espinoza LR. IgG4-associated vasculitis. Curr Rheumatol Rep 2013; 15: 348. 